\title{
Spinal cord herniation is one of the important differential diagnoses of spinal arachnoid cyst
}

\author{
Takeo Baba • Izumi Koyanagi
}

Received: 16 November 2010 / Accepted: 19 November 2010/Published online: 16 December 2010

(C) Springer-Verlag 2010

We greatly appreciate the comment by Drs. Groen and Coopes on our recent article, "Pulsatile wall movement of spinal arachnoid cyst deteriorates spinal cord symptoms: report of three cases". Spinal cord herniation is one of the important differential diagnoses of spinal arachnoid cyst. From our experience, spinal cord herniation can be visualized by thin slice MRI or CT myelography. The spinal cord is attached to the ventral dura mater with localized cord deformity. Clinically, the patients with spinal cord herniation often showed the Brown-Séquard syndrome with several years of history.

In our three cases, the patients presented with a rather rapid progression of symptoms, and MRI showed mild ventral displacement of the spinal cord. Thin slice axial scans did not disclose the localized cord deformity (herniated part of the spinal cord). In our patients, the pulsatile cerebrospinal fluid movement, especially rostral flow during the diastolic phase of the cardiac cycle, caused spinal cord compression by the parachute-like expansion of the arachnoid cyst. Such dynamic pathophysiology could be appreciated with cine-mode MRI and intraoperative ultrasonography. During surgery, we confirmed the absence of the ventral dural defect in all three cases.

We quite agree with Drs. Groen and Coopes that clinicians should be aware of the possibility of spinal cord herniation in cases of ventral spinal cord displacement. However, the possibility of the arachnoid cyst with a dynamic compressive pathomechanism will also be important when MRI shows only mild spinal cord displacement.

Conflicts of interest None.

\footnotetext{
T. Baba $(\bowtie)$

Department of Neurosurgery,

Shinsapporo Neurosurgical Hospital,

1-2-1-10 Kaminopporo, Atsubetu-ku,

Sapporo 004-0031, Hokkaido, Japan

e-mail: baba@snh.or.jp

I. Koyanagi

Department of Neurosurgery, School of Medicine, Sapporo

Medical University,

Sapporo, Hokkaido, Japan
} 